\title{
Las tecnologías digitales emergentes entran en la Universidad: RA y RV
}

\section{Emerging digital technologies come into the University: AR and VR}

\author{
Julio Cabero Almenara \\ Universidad Sevilla (España) \\ Bárbara Fernández Robles \\ Universidad Isabel I (España)
}

\section{Resumen}

En la última década están emergiendo un conjunto de tecnologías que se están acercando a la educación universitaria como no había sucedido en épocas anteriores. Muchas de estas tecnologías pueden ser consideradas como disruptivas, puesto que están transformando y mejorando los escenarios formativos. Entre las tecnologías que están tomando mayor impulso e importancia encontramos la realidad aumentada y la realidad virtual, tal y como han puesto de manifiesto diversos informes Horizon y Edutrens. A pesar de que las investigaciones sobre la integración de estas tecnologías se encuentran en un estado incipiente, podemos afirmar que las realizadas hasta el momento demuestran los grandes beneficios que aportan al proceso de enseñanza-aprendizaje. Entre estas contribuciones podemos destacar la mejora de la motivación, de la satisfacción y del rendimiento de los alumnos. En el presente artículo mostramos el estado actual de la Universidad en lo que a integración de TIC respecta, haciendo alusión a las tecnologías que están irrumpiendo con mayor fuerza en los últimos tiempos. Además nos centramos en dos de las tecnologías "realidad aumentada y realidad virtual" que están teniendo mayor repercusión en los últimos años, demostrando evidencias al respecto y haciendo mención a su incorporación en el ámbito universitario.

Palabras claves: tecnologías de la información y de la comunicación; cambio tecnológico; universidad; realidad aumentada; realidad virtual.

\begin{abstract}
A set of emerging technologies have been approaching university education in the last decade as it had not happened in previous times. Many of these technologies can be considered as disruptive, since they are transforming and improving the training scenarios. Among the technologies that are taking greater momentum and importance we find augmented reality and virtual reality, as evidenced by various reports Horizon and Edutrens. Although the research on the integration of these technologies is in an early stage, we can affirm that those carried out so far demonstrate the great benefits they incorporate into the teaching-learning
\end{abstract}


process. Among these contributions we can highlight the improvement of the motivation, the satisfaction and the performance of the students. In this article, we will cover the current state of the University regarding ICT integration, focusing on the technologies that have been bursting with greater force in recent times. In addition, we will focus on two of the technologies "augmented reality and virtual reality" that are experiencing more impact in recent years, showing evidences and mentioning their implementation in the university environment.

Keywords: information and communication technologies; technological change; university; augmented reality; virtual reality.

Las universidades han estado generalmente muy comprometidas con la penetración de las Tecnologías de la Información y Comunicación (TIC) en las actividades de gestión, investigación y docencia que realizan; es más, podríamos decir que han sido el verdadero espacio de innovación y experimentación para su incorporación. Tecnologías como la formación virtual (Marciniak y Gairín, 2017), e-portafolios (Cabero, López y Jaén, 2013), MOOC (Gómez, 2014), dispositivos móviles (Merino, Cabello y Merino, 2017), los entornos 3D (Esteve-González, González, Gisbert y Cela, 2017), o la realidad aumentada (Marín, 2017), han sido incorporadas inicialmente en la formación universitaria y de este se ha extendido a otros contextos.

Tal grado de penetración podemos observarla en los diferentes informes Universitic que el Consejo de Rectores de las Universidades Españolas publican desde el año 2007. Así en el último se destaca:

Las universidades utilizan un $87 \%$ de los servicios de soporte TIC a la docencia incluidos en el catálogo. La docencia virtual, la gestión de licencias software para docencia y el soporte a aulas de informática de uso docente están implantados prácticamente en la totalidad de las universidades (Gómez, 2016, p.12).

En este mismo informe Gómez (2016, p. 13) destaca que "los servicios y las infraestructuras TI para la docencia presencial y la no presencial ya no están diferenciados, al haber evolucionado la docencia presencial hacia una fuerte utilización de las tecnologías educativas”.

Esta situación ha llevado a algunos autores a señalar que la Universidad actual se siente transformada por diferentes tensiones: la globalización, las características de los nuevos alumnos, la internacionalización, la competencia entre universidades por la captación de estudiantes y las TIC. Lo comentado repercutirá en una serie de desafíos para las universidades, que irán desde las nuevas competencias que deben poseer los docentes, las nuevas competencias que se deben desarrollar en el currículum, y la contemplación de nuevos diseños instruccionales más acordes con los nuevos escenarios tecnológicos. 
Para que la institución universitaria pueda asumir su función esencial en la generación de innovación tiene que transformarse a sí misma, tiene que ser Universidad innovadora. Tiene que integrar la enseñanza presencial y virtual en un continuo interactivo que pueda aprovechar las oportunidades que ofrece el entorno digital sin enterrar la tradición magisterial y el contacto directo en el aula. Tiene que articularse fuertemente con el entorno empresarial, así como con la aplicación de sus conocimientos en las instituciones de servicios públicos. Tiene que escapar de la dicotomía destructiva entre la universidad para las élites y el café para todos que acaba no siendo café (Castell, 2009, p. 50).

Por su parte, en el último informe Horizon sobre Universidades (Adams et al., 2017), se señalan diferentes transformaciones que estas instituciones de formación deben abordar en los momentos actuales, y que son: potenciación del emprendimiento y el descubrimiento, transformación en los enfoques de aprendizaje, crear grupos centrados en la medición del aprendizaje, rediseño de los espacios de aprendizaje, diseño de aprendizajes mezclados y apoyo en el aprendizaje colaborativo.

En estos nuevos escenarios las TIC desempeñarán un papel significativo por varios motivos: extender sus espacios de influencia, ampliar la formación fuera de los espacios tradicionalmente reglados, flexibilizar los escenarios de formación, y potenciar los escenarios enriquecidos por las tecnologías. Igualmente, no podemos olvidarnos de ofrecer amplia información y realizar prácticas hoy día impensables sin el apoyo de tecnologías, como por ejemplo los entornos de simulación.

Ante este panorama, es necesario conocer de cerca las tendencias tecnológicas que se acercan al sistema universitario con el fin de crear conocimiento sobre las potencialidades para la formación y sobre la toma de decisiones para su integración en el terreno educativo.

Con este objetivo surge el presente estudio, con el que se pretende indagar sobre las tecnologías que están impactando con mayor fuerza en este nivel educativo, y en concreto, sobre la repercusión que están teniendo en la universidad la realidad aumentada y la realidad virtual.

\section{TECNOLOGÍAS EMERGENTES APLICADAS A LA FORMACIÓN}

En la última década han aparecido una diversidad de tecnologías que se han acercado al terreno educativo como no había ocurrido en las décadas anteriores, en las cuales las tecnologías se habían mantenido más o menos estables, alrededor de lo audiovisual, auditivo, informático y multimedia.

Sin embargo, en los últimos tiempos están introduciéndose en las instituciones educativas un fuerte volumen de tecnologías novedosas, algunas de las cuales se caracterizan como disruptivas, que como apunta Christensen (2012) son aquellas que mejoran un producto o servicio de una manera no esperada por el mercado y que en consecuencia rompen radicalmente con lo existente. 
Tales tecnologías pueden ser conocidas a través de diferentes propuestas, una de ellas es la de los Informes Horizon que realiza la Institución "NMC" https://www. nmc.org/nmc-horizon, y que persiguen analizar las tecnologías que al horizonte de un año, tres o cinco, se percibe que se incorporarán a los diferentes niveles de la institución educativa. Por lo que se refiere a la educación universitaria se han realizado diversos, y en la tabla 1 se presentan las propuestas realizadas.

Tabla 1. Propuestas de tecnologías emergentes de acuerdo a diferentes proyectos Horizon

\begin{tabular}{|c|c|c|c|}
\hline Informes Horizon & A 1 año 0 menos & De 2 a 3 años & De 3 a 6 años \\
\hline $\begin{array}{l}\text { Durall, Gros, Maina, } \\
\text { Johnson y Adams } \\
\text { (2012). }\end{array}$ & $\begin{array}{l}\text { Contenidos abiertos } \\
\text { Aplicaciones móviles } \\
\text { Computación en } \\
\text { nubes } \\
\text { Entornos } \\
\text { colaborativos }\end{array}$ & $\begin{array}{l}\text { Tabletas } \\
\text { Aprendizaje basado } \\
\text { en juegos } \\
\text { PLE } \\
\text { Geolocalización }\end{array}$ & $\begin{array}{l}\text { Analíticas de } \\
\text { aprendizaje } \\
\text { Realidad aumentada } \\
\text { Web Semántica } \\
\text { MOOC }\end{array}$ \\
\hline Johnson et al. (2013) & $\begin{array}{l}\text { Contenidos Abiertos } \\
\text { Aprendizaje en línea } \\
\text { Entornos } \\
\text { colaborativos } \\
\text { Medios sociales }\end{array}$ & $\begin{array}{l}\text { Realidad Aumentada } \\
\text { Aprendizaje móvil } \\
\text { Aprendizaje } \\
\text { personalizado } \\
\text { Analíticas de } \\
\text { aprendizaje } \\
\end{array}$ & $\begin{array}{l}\text { Aprendizaje } \\
\text { aumentado } \\
\text { Impresión en 3D } \\
\text { Internet de las cosas } \\
\text { Laboratorios } \\
\text { virtuales y remotos }\end{array}$ \\
\hline $\begin{array}{l}\text { Johnson, Adams, } \\
\text { Estrada y Freeman } \\
\text { (2014) }\end{array}$ & $\begin{array}{l}\text { Flipped Classroom } \\
\text { Analíticas de } \\
\text { aprendizaje }\end{array}$ & $\begin{array}{l}\text { Impresión } 3 \mathrm{D} \\
\text { Videojuegos y } \\
\text { gamificación }\end{array}$ & $\begin{array}{l}\text { Quantified self } \\
\text { Asistentes virtuales }\end{array}$ \\
\hline Johnson et al. (2016). & $\begin{array}{l}\text { Trae tu propio } \\
\text { dispositivo } \\
\text { Analíticas de } \\
\text { aprendizaje }\end{array}$ & $\begin{array}{l}\text { Realidad Aumentada } \\
\text { y virtual } \\
\text { Talleres creativos }\end{array}$ & $\begin{array}{l}\text { Computación afectiva } \\
\text { Robótica }\end{array}$ \\
\hline Adams et al. (2017) & $\begin{array}{l}\text { Tecnologías } \\
\text { de aprendizaje } \\
\text { adaptativo. } \\
\text { Aprendizaje móvil }\end{array}$ & $\begin{array}{l}\text { Internet de las cosas } \\
\text { La próxima } \\
\text { generación de LMS }\end{array}$ & $\begin{array}{l}\text { Inteligencia artificial } \\
\text { La interfaz natural de } \\
\text { usuario }\end{array}$ \\
\hline
\end{tabular}

Como puede observarse, las propuestas tecnológicas que se han efectuado son diferentes y variadas, y algunas de estas, si miramos nuestras aulas, nos daremos cuenta que se han incorporado hace algunos años, como por ejemplo la política de los contenidos abiertos, las aplicaciones móviles, o los PLE.

Por otra parte, si revisamos los reportes EduTrends elaborados por el Observatorio de Innovación Educativa del Instituto Tecnológico de Monterrey (2015, 2016 y 2017), referidos a las tendencias tecnológicas que impactarían a corto plazo en la institución educativa citada, nos encontramos también con una amplia gama tecnológica tal y como muestra la tabla 2. 
Tabla 2. Propuestas de tecnologías emergentes de acuerdo a diferentes Informes EduTrends

\begin{tabular}{|l|l|}
\hline \multicolumn{1}{|c|}{ Informes } & \multicolumn{1}{|c|}{ Tecnologías } \\
\hline EduTrçends (2015) & $\begin{array}{l}\text { Aprendizaje adaptativo } \\
\text { Entornos personalizados de aprendizaje } \\
\text { Aprendizaje ubicuo } \\
\text { Internet de las cosas } \\
\text { Laboratorios remotos y virtuales } \\
\text { Realidad aumentada }\end{array}$ \\
\hline EduTrends (2016) & $\begin{array}{l}\text { Aprendizaje adaptativo } \\
\text { Aprendizaje en redes sociales y entornos colaborativos } \\
\text { Entornos personales de aprendizaje } \\
\text { Insignias y microcréditos } \\
\text { Realidad Aumentada }\end{array}$ \\
\hline EduTrends (2017) & $\begin{array}{l}\text { Aprendizaje adaptativo } \\
\text { Aprendizaje en redes sociales y entornos colaborativos } \\
\text { Aprendizaje móvil } \\
\text { Big data y Analíticas de aprendizaje } \\
\text { MOOC. }\end{array}$ \\
\hline
\end{tabular}

Finalmente, para presentar las diferentes tecnologías emergentes que están acercándose al terreno educativo universitario, indicaremos la propuesta que anualmente presenta la empresa Gartner con su conocido hiperciclo donde muestran la significación y evolución adquirida por diferentes tecnologías. De acuerdo con su modelo de análisis tecnológico, toda tecnología pasa por diferentes etapas: lanzamiento (Technology Trigger), pico de expectativas sobredimensionadas (Peak of Inflated Expectations), abismo de desilusión (Trough of Disillusionment), rampa de consolidación (Slope of Enlightenment), y meseta de productividad (Plateau of Productivity). Siendo en la última donde la tecnología se consolida y se desprende de todas sus sobredimensiones. En la figura 1, se presenta la propuesta realizada en 2017. 
Figura 1. Hiperciclo de Gartner para 2017

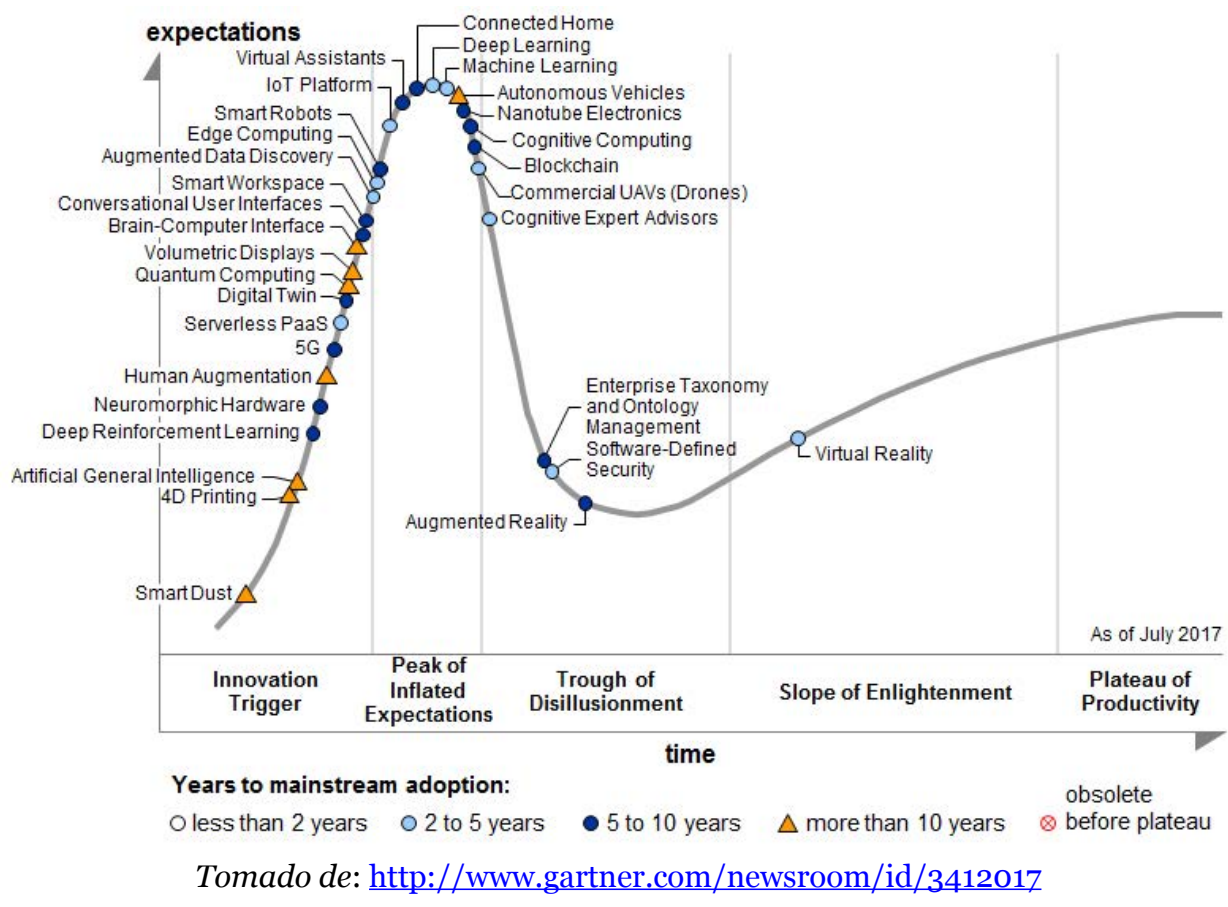

El análisis de las diferentes propuestas realizadas nos lleva a indicar un cúmulo de tecnologías emergentes que están acercándose en los últimos tiempos al terreno educativo, que van desde los MOOC, las redes sociales, las analíticas de aprendizaje, la internet de las cosas, la web semántica, los entornos personales de aprendizaje, o la realidad aumentada y virtual, centrándonos a partir de este momento en las dos últimas.

\section{REALIDAD AUMENTADA Y REALIDAD VIRTUAL}

Como se ha visto en el apartado anterior, tanto la realidad aumentada (RA) como virtual (RV), son tecnologías que están penetrando últimamente en la Universidad y están facilitando el acercamiento de esta institución hacia su digitalización. El aumento de su significación podemos observarlo al incorporar ambos términos al programa "Ngram Vieewer" de Google que facilita conocer el aumento de dichos términos en diferentes publicaciones (figura 2). 
Figura 2. Evolución de los términos "augmented reality" y "virtual reality" de acuerdo al programa "Ngram Vieewer"
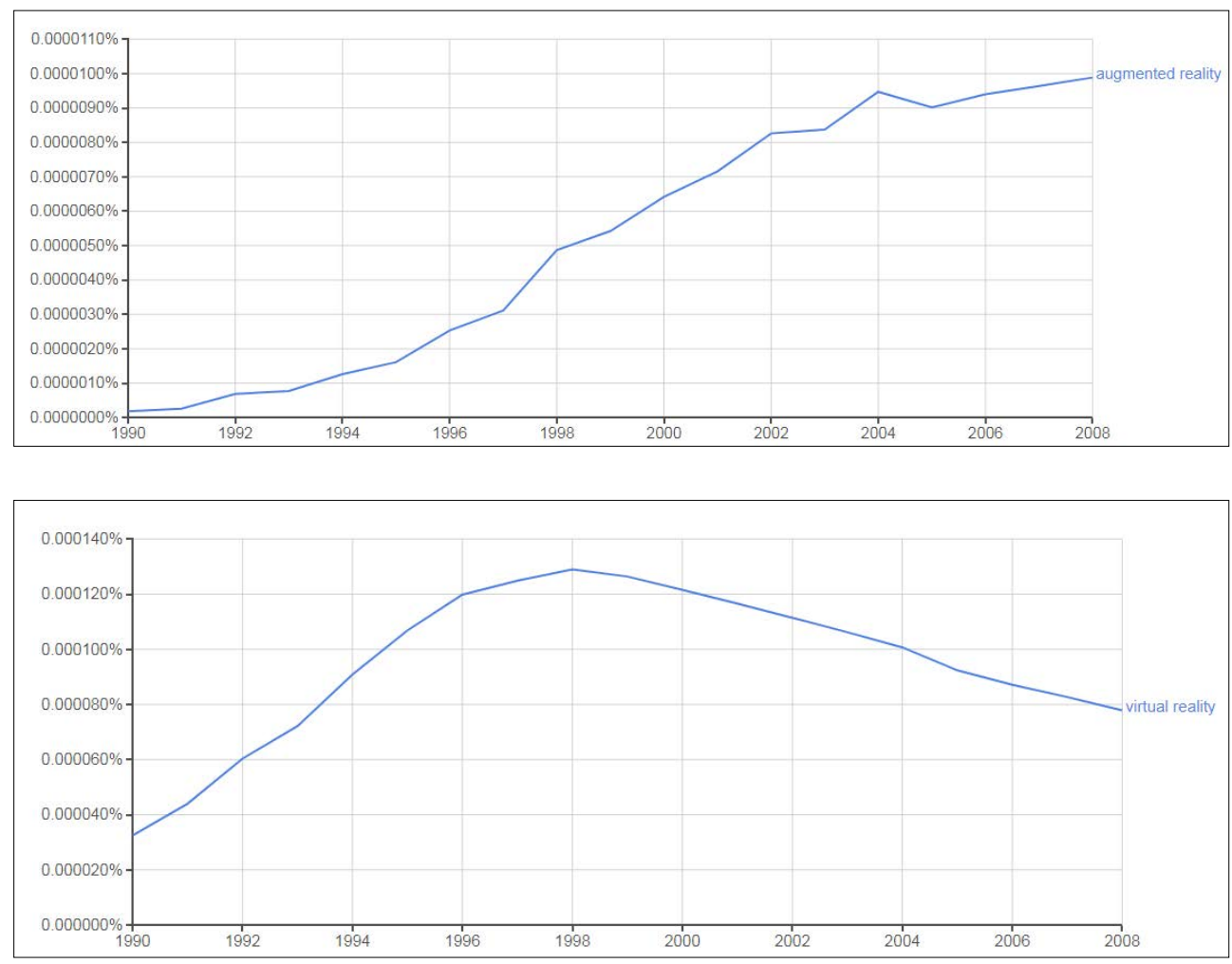

Como se puede observar ambos términos, aunque uno más que otro posiblemente por la dificultad que implica su producción y la necesidad de dispositivos especiales para su observación como ocurre con la RV, tienen una presencia y evolución significativa. Y como se desprende de los ciclos de sobreexpectación de Gartner (figura 1) son tecnologías que ya empiezan a adentrarse en la meseta de la productividad, que es el momento en el cual las tecnologías se generalizan en la sociedad y adquieren aplicabilidad y relevancia en los mercados e instituciones.

Es el momento ahora de determinar qué es lo que se entiende por RA y RV. Para ello podemos apoyarnos en la propuesta que realizan Milgram y Kishino (1994) en el continuum realidad-virtualidad (figura 3). 
Figura 3. Continuum realidad-virtualidad

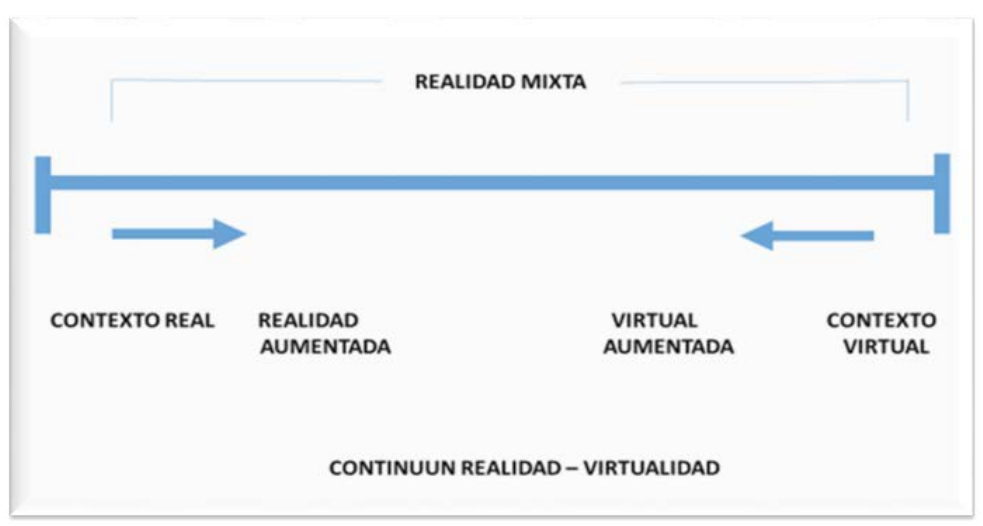

Tomado de: Milgram y Kishino, 1994

Como puede observarse en este continuum, la RA estaría más cerca del contexto real, mientras que la RV se ubicaría en uno de los extremos, estando en el centro la "Realidad Virtual Aumentada" o "Realidad Mixta"; tecnología esta última que incorpora elementos de la RA y RV, y que en los últimos tiempos se está extendiendo.

Otra manera de caracterizarlas es considerar que en la RA se combina la realidad con elementos informativos disponibles en los dispositivos tecnológicos utilizados para conseguir una nueva realidad, mientras que en la $\mathrm{RV}$, la persona se sitúa en un entorno inmersivo tecnológico, y por tanto creado artificialmente, y no se mezcla de esta forma con la realidad (Díaz, 2016; Johnson y Adams, 2016). En la primera se mezcla la realidad con lo virtual, y en la segunda lo que se propicia es la ubicación del sujeto en un mundo alternativo creado artificialmente a través de los ordenadores y donde ocurren diferentes tipos de experiencias sensoriales.

Como señala Brigham (2017), la diferencia fundamental estriba en que en la RA el sujeto se mantiene en su espacio, mientras que, en el caso de la RV, la persona se desplaza a otro lugar; en definitiva, podríamos decir que en el primero el sujeto se encuentra localizado en su contexto real, mientras que con la segunda se deslocaliza del mismo.

Cabero y García (2016, p. 7) señalan que la RA "trata de una tecnología que permite la combinación de información digital e información física en tiempo real por medio de distintos soportes tecnológicos como por ejemplo las tabletas o los smartphones, para crear con ello una nueva realidad enriquecida".

Por su parte Brudniy y Demilhanova (2012, p. 6) llaman la atención respecto a que la RV es:

La forma más avanzada de relación entre una persona y un sistema informático, dicha relación permite una interacción directa entre el usuario y el ambiente generado 
artificialmente, ambiente que está destinado a estimular alguno o todos los sentidos humanos, caracterizándose principalmente por crear una ilusión a nivel cerebral de participación directa en dicho ambiente.

A esta última definición podríamos incorporarle el matiz indicado por Cañellas (2017) para quién la RV:

Es aquella tecnología que posibilita al usuario, mediante el uso de un visor RV, sumergirse en escenarios virtuales, en primera persona y en $360^{\circ}$. Esto propicia que el usuario se sienta inmerso dentro de dichos escenarios, e incluso que pueda interactuar con los elementos que los componen. El usuario deja de ver el lugar en el que se encuentra ubicado, para pasar a visualizar e interactuar con otra realidad.

La separación entre ambas tecnologías, podemos también comprenderla a partir de los diferentes niveles que cada una permite. En la RA se pueden diferenciar cinco niveles basados en diferentes elementos, que son utilizados como lanzadores de la información: un patrón artificial en blanco y negro o código QR, una imagen, un objeto $3 \mathrm{D}$, un punto del planeta determinado por sus coordenadas GPS, y huella termal (Cabero y García, 2016).

Por lo que se refiere a la RV nos encontramos con sistemas de escritorio o no inmersivo, semi-inmersivo y de inmersión total (Flores, Camarena y Avalos, 2014); aunque algunos autores (Cañellas, 2017) solamente consideran dos tipos: inmersivos, basados en la simulación de escenarios 2D o 3D, en los que el usuario percibe dichos escenarios con sensación de "presencialidad" en primera persona, como si estuviera realmente inmerso dentro, y no inmersivos, basados en la visualización de escenarios virtuales a través de una pantalla, dando la sensación a la persona de estar mirando el escenario virtual desde una "ventana".

\section{METODOLOGÍA}

La necesidad de crear conocimiento sobre las tendencias tecnológicas en el ámbito universitario hizo que pusiésemos en marcha una investigación documental sobre tecnologías emergentes en el ámbito universitario. Con la misma pretendíamos buscar, recuperar, analizar e interpretar datos obtenidos y registrados por otros estudios e investigaciones (Sabino, 2006), con el fin de conocer el estado en el que se encuentran algunas tecnologías emergentes y definir nuevas líneas de investigación y/o actividades relacionadas con la integración de TIC en el ámbito universitario.

Para comenzar el estudio se revisaron los últimos informes Horizon, informes Edutrens y el Hiperciclo de la empresa Gartner. Tras lo cual se encontró que la realidad aumentada y la realidad virtual son dos de las tecnologías que están impactando con mayor fuerza en educación en los últimos años, por lo que decidimos centrarnos en indagar sobre las investigaciones llevadas a cabo sobre estas tecnologías en el ámbito educativo. 
Una vez tomada esta decisión, realizamos una revisión bibliográfica sobre estudios que tratasen el uso e integración de estas tecnologías en educación. Finalmente, se hizo una revisión de la producción científica de 45 revistas entre los años 2010 y 2017.

Tras esta revisión se seleccionaron 28 de las revistas científicas revisadas, siendo el motivo principal para elegir estas revistas el hecho de que recogían artículos sobre realidad aumentada y/o realidad virtual, lo cual fue complejo, debido al estado incipiente en el que se encuentran las investigaciones sobre el uso didáctico de ambas tecnologías.

Seguidamente mostramos las revistas y el número de artículos seleccionados para realizar el estudio.

Tabla 3. Revistas y número de artículos seleccionados

\begin{tabular}{|l|l|}
\hline \multicolumn{1}{|c|}{ Revistas seleccionadas } & $\begin{array}{c}\text { Número de } \\
\text { artículos } \\
\text { extraídos }\end{array}$ \\
\hline Apertura. Revista de Innovación Educativa & 1 \\
\hline Australasian Journal of Educational Technology & 2 \\
\hline Automation in Construction & 1 \\
\hline Behaviour \& Information Technology & 1 \\
\hline British Journal of Educational Technology & 3 \\
\hline Canadian Medical Education Journal & 1 \\
\hline Computers in Human Behavior & 2 \\
\hline Computers \& Education & 7 \\
\hline Education in the Knowledge Society & 1 \\
\hline Education Technology Research Development & 1 \\
\hline Educational Technology y Society & 1 \\
\hline Edmetic & 1 \\
\hline Educational Media International & 1 \\
\hline Enseñanza \& Teaching & 1 \\
\hline Formación Universitaria & 1 \\
\hline iJET & 2 \\
\hline International Education Studies & 1 \\
\hline International Journal of Emerging Technologies in Learning & 1 \\
\hline Journal of Educational Computing Research & 1 \\
\hline Mobile Media \& Communication & 1 \\
\hline NorDina & 1 \\
\hline Quid & 1 \\
\hline RED. Revista de Educación a Distancia & 1 \\
\hline & \\
\hline
\end{tabular}




\begin{tabular}{|l|l|}
\hline \multicolumn{1}{|c|}{ Revistas seleccionadas } & $\begin{array}{c}\text { Número de } \\
\text { artículos } \\
\text { extraídos }\end{array}$ \\
\hline Research and Practice in Technology Enhanced Learning & 2 \\
\hline $\begin{array}{l}\text { Revista Interuniversitaria de Investigación en Tecnología } \\
\text { Educativa (RIITE) }\end{array}$ & 1 \\
\hline Ventana Informática & 1 \\
\hline World Journal on Educational Technology: Current Issues & 1 \\
\hline
\end{tabular}

En total escogimos 40 artículos relacionados con investigaciones y estudios puestos en marcha sobre estas tecnologías. Además, se revisaron otras fuentes como libros y conferencias, encontrando que había algunos elementos en los que varios autores hacían alusión de estas tecnologías, los cuales pasamos a presentar seguidamente.

\section{RESULTADOS}

En este apartado profundizamos en algunos aspectos que se ponen de relieve en la literatura científica revisada sobre la RA y la RV. Para ello, vamos a presentar una tabla en la que planteamos algunos elementos comunes que comparten varios autores, en la columna izquierda queda recogida la afirmación realizada por varios estudios y en la columna derecha los autores que la han realizado.

Tabla 4. Análisis efectuados

\begin{tabular}{|l|l|}
\hline \multicolumn{1}{|c|}{ Afirmación realizada } & \multicolumn{1}{c|}{ Autores } \\
\hline Falta de investigaciones. & $\begin{array}{l}\text { Alkhattabi, 2017; Bacca, Bower, Howe, } \\
\text { McCredie, Robinson y Grover, 2014; Cheng y } \\
\text { Tsai, 2013; Di Serio, Ibañez y Delgado, 2013; } \\
\text { Kim y Ke, 2016; Lin, Been-Lirn, Wang y } \\
\text { Tsa, 2013; Nielsen, Brandt y Swensen, 2016; } \\
\text { Saidin, Halim y Yahaya, 2015; Wang, Jeong, } \\
\text { Love y Kang, 2013; Wu, Chang y Liang, 2013 }\end{array}$ \\
\hline $\begin{array}{l}\text { Falta de modelos conceptuales para su } \\
\text { incorporación. }\end{array}$ & $\begin{array}{l}\text { Bower et al., 2014; Rasimah, Ahmad y } \\
\text { Zaman, 2011 }\end{array}$ \\
\hline $\begin{array}{l}\text { Últimamente se están formulando } \\
\text { propuestas a partir de la combinación de } \\
\text { diferentes enfoques pedagógicos y teorías de } \\
\text { aprendizaje. }\end{array}$ & $\begin{array}{l}\text { Bower et al., 2014; Fowler, 2015; Rasimah et } \\
\text { al., 2011. }\end{array}$ \\
\hline \multirow{2}{*}{$\begin{array}{l}\text { Despierta altos niveles de satisfacción. } \\
\text { Cabero, García y Arroyo, 2016; Fonseca, } \\
\text { Redondo y Valls, 2016; Han, Jo, Hyun y So, } \\
\text { 2015; Hsu, Lin y Yang, 2017; Pérez-López, } \\
\text { 2015 }\end{array}$} \\
\hline
\end{tabular}


Afirmación realizada

La motivación aumenta con su uso.

Favorece el desarrollo de la inteligencia espacial.

Los apuntes enriquecidos en RA aumentan la motivación, la atención y el rendimiento.

La RV aumenta la creatividad.

\section{Autores}

Barroso y Cabero, 2016; Bicen y Bal, 2016; Cantón, Arellano, Hernández y Nieva, 2017; Chiang, Yang y Hwang, 2014; Cheng, 2017; Nielsen et al., 2016

Alvarez, Castillo, Pizarro y Espinoza, 2017; Carbonnel y Bermejo, 2017; Del Cerro Velázquez y Morales-Méndez, 2017; Guo, 2016; Kim y Ke, 2016.

Cheng, 2017; Martín-Gutiérrez, Fabiani, Bensova y Meneses, 2015; Nadolny, 2016 Chang, Zhang y Jin, 2016; Jimeno-

Morenilla, Sánchez-Romero, Mora-Mora y Collo-Miralles, 2016

Chang et al., 2016; Reinoso, 2016

\section{CONCLUSIÓN}

Sin dejar de reconocer que uno de los problemas que tenemos actualmente para la incorporación de estas tecnologías es la falta de investigaciones (Alkhattabi, 2017; Bacca, Baldiris, Fabregat, Graf y Kinshuk, 2014; Bates, 2017; Bower, Howe, McCredie, Robinson y Grover, 2014; Cheng y Tsai, 2013; Di Serio, Ibañez y Delgado, 2013; Kim y Ke, 2016; Lin, Been-Lirn, Wang y Tsa, 2013; Nielsen, Brandt y Swensen, 2016; Saidin, Halim y Yahaya, 2015; Wang, Jeong, Love y Kang, 2013; Wu, Chang y Liang, 2013) y de modelos conceptuales para su incorporación a la enseñanza (Bower et al., 2014; Rasimah, Ahmad y Zaman, 2011), debemos asumir que últimamente se están formulando propuestas a partir de la combinación de diferentes enfoques pedagógicos y teorías de aprendizaje, tales como: el constructivista, experiencial, basado en problemas, situado, inductivo, y el basado en juegos (Bower et al., 2014; Fowler, 2015; Rasimah et al., 2011; Tarng, y Ou, 2012). Dichos estudios están apuntando principios tanto para el diseño de los objetos de aprendizaje como para la búsqueda de estrategias de utilización.

Por lo comentado, tenemos que reconocer que en los últimos tiempos se han producido un cúmulo de trabajos que nos permiten tener referencias, tanto para su incorporación a la enseñanza, como para las formas en las cuales se pueden diseñar sus mensajes, y las estrategias educativas que podemos movilizar en su utilización.

Un volumen de investigaciones se ha centrado en analizar el grado de satisfacción que despierta su utilización en los estudiantes, y en este aspecto los resultados encontrados van en las siguientes direcciones: los estudiantes revelan altos niveles de satisfacción cuando participan en experiencias de este tipo, los resultados son similares con ambas tecnologías, los hallazgos son independientes del género de los estudiantes y de los estudios en los cuales se realizaron (Cabero et al., 2016; Fonseca 
et al., 2016; Han et al., 2015; Hsu et al., 2017; Pérez-López, 2015; Rodríguez, Naranjo y Duque, 2016).

Al mismo tiempo, las experiencias realizadas indican el aumento de la motivación de los estudiantes, tanto hacia los contenidos como hacia la formación recibida, cuando participan en experiencias formativas con RA y/o RV (Barba, Yasaca y Manosalvas, 2015; Barroso y Cabero, 2016; Bicen y Bal, 2016; Cantón et al., 2017; Chiang et al., 2014; Cheng, 2017; Freina y Ott, 2015; Nielsen et al., 2016).

Ello es explicado por diferentes motivos, que van desde la posibilidad que ofrecen para representar los contenidos de diversas formas, los entornos de simulación inmersivos que se crean, el enriquecer los libros y apuntes con documentos audiovisuales y multimedia, el concretar la información, y el que permite que los estudiantes interaccionen y manipulen los objetos.

Otro grupo de investigaciones han obtenido como resultado el que su utilización favorece el desarrollo dela inteligencia espacial, la orientación espacial, las habilidades espaciales de la persona y la comprensión visual de los objetos y contextos. Estos resultados se han presentado tanto cuando los alumnos interaccionan con objetos de RA (Alvarez et al., 2017; Carbonnel y Bermejo, 2017; Del Cerro Velázquez y MoralesMéndez, 2017; Lee, Chen y Chang, 2016) como con los de RV (Guo, 2016; Hwang y $\mathrm{Hu}, 2013$; Kim y Ke, 2016).

Por lo que se refiere al rendimiento académico, los resultados no son tan concluyentes como cabría esperar por las posibilidades que se le han concedido a ambas tecnologías, y oscilan entre aquellos que han encontrado resultados positivos y significativos (Alvarez et al., 2017; Lin et al., 2013; Pedraza y Valbuena, 2014; Santos et al., 2016), y los que indican que no se ve afectado o que el aprendizaje disminuye (Hofmann y Mosemghvdlishvili, 2014; Pérez-López, 2014). En este aspecto debemos reconocer que uno de los errores cometidos en diversos estudios, sobre todo en los que se han obtenido resultados significativos, es la no contemplación del efecto novedad (Cabero, 2016).

Las investigaciones que no encontraron resultados significativos respecto al rendimiento lo explicaron por las siguientes causas: las características de las aplicaciones RA en los teléfonos móviles disponibles en la actualidad ya que algunas de ellas requerían gran capacidad, la conectividad wifi existente en las estancias educativas, la desorientación cognitiva que sufren ciertos sujetos al interaccionar con estas tecnologías, la sensación de mareo que producen ciertos dispositivos de observación en el caso de la RV, o que el sujeto se siente fascinado por el entorno tecnológico y se olvida de la acción formativa.

Específicamente, y por lo que se refiere a la RA utilizada bajo la forma de libros y apuntes enriquecidos con esta tecnología, los resultados encontrados apuntan un aumento de la motivación, el desarrollo de actitudes positivas hacia la enseñanza y la mejora del rendimiento académico (Cheng, 2017; Ferrer, Jiménez, Torralba y García, 2016; Martín-Gutiérrez et al., 2015; Nadolny, 2016).

Debemos también resaltar los comentarios realizados por Cuendet, Bonnard, Do-Lenh y Dillenbourg (2013), quienes señalan que para que la RA tenga una 
utilización eficaz debe reunir las siguientes condiciones: que el sistema creado sea lo suficientemente flexible para que el profesor lo adapte a las necesidades de sus estudiantes, y que el sistema elaborado tenga en cuenta las restricciones, sobre todo tecnológicas, presentes en el contexto educativo donde se utiliza.

En el caso de la RV tenemos que señalar que las aplicaciones más significativas se han desarrollado bajo la modalidad de elaborar con ella contextos y entornos de simulación (Flores, Camarena y Avalos, 2014; Walsh et al., 2017), aunque Flores et al. (2014) amplían los usos a los de: modelar (partículas, ambientes físicos, y características humanas), representación de la realidad (objetos, situaciones y personas), y diseñar (espacios, productos, herramientas y prototipos).

Por su parte Bates (2017) respecto a la RV señala que: 1) no es solo una moda, ya hay un gran número de aplicaciones comerciales y en áreas específicas de formación; 2) el costo del hardware está cayendo rápidamente (aunque los auriculares de buena calidad y otros equipos son probablemente demasiado caros para el uso requerido por un gran número de estudiantes); 3) lo que mató los desarrollos de RV bidimensionales anteriores como Second Life para uso educativo generalizado fue el alto costo y la dificultad de crear los conjuntos y contextos para el aprendizaje; 4) y que es probable que las aplicaciones educativas más adecuadas sean donde el costo de las formas alternativas o tradicionales de aprendizaje es demasiado costoso o demasiado peligroso. En particular, sería buena para el autoaprendizaje de la persona en contextos en los que los entornos reales no son fácilmente accesibles, $o$ donde los estudiantes necesitan hacer frente a emociones fuertes al tomar decisiones u operar bajo presión en tiempo real.

Conviene señalar también que dentro de los usos educativos de la RA y RV se empieza a contemplar la posibilidad que ofrecen en situaciones de enseñanza a distancia, de teleformación y e-learning (Chang et al., 2016; Reinoso, 2016).

No podemos olvidar que ambas tecnologías requieren competencias digitales diferentes para la producción de objetos; así la RA se puede producir con mayor facilidad que la RV. Tal es su grado de facilidad que ya existen experiencias de producción de estos objetos, tanto por los docentes como por los discentes (Barroso y Gallego, 2017).

Para finalizar, se quiere resaltar que diferentes hechos y acontecimientos están repercutiendo para que sea una tecnología que está penetrando en la formación universitaria, como son: la producción de objetos de aprendizaje http://ra.sav.us.es; http://ra.sav.us.es/index.php?option=com content\&view=article\&id=105\&Itemid=175), la realización de eventos y congresos como el Aumentame (http://aumenta.med), la aparición de monográficos en revistas sobre la temática (como el volumen 6 número 1 de la revista Edmetic denominado aplicaciones de la Realidad Aumentada en la Educación - http://www. uco.es/ucopress/ojs/index.php/edmetic/issue/view/546/showTod, o la realización de proyectos de investigación I+D+i como el denominado: "Realidad Aumentada para Aumentar la Formación. Diseño, Producción y Evaluación de Programas de 
Realidad Aumentada para la Formación Universitaria (RAFODIUN) (EDU201457446-P)" http://grupotecnologiaeducativa.es/proyectorafodiund).

\section{REFERENCIAS BIBLIOGRÁFICAS}

Adams, S., Cummins, M., Davis, A., Freeman, A., Hall, C., y Ananthanarayanan, V. (2017). NMC Horizon Report: 2017 Higher Education Edition. Austin: The New Media Consortium.

Alkhattabi, M. (2017). Augmented Reality as E-learning Tool in Primary Schools' Education: Barriers to Teachers' Adoption. International Journal of Emerging Technologies in Learning, 12(2), 91-100.

Alvarez, A., Castillo, M., Pizarro, J., y Espinoza, E. (2017). Realidad Aumentada como Apoyo a la Formación de Ingenieros Industriales. Formación Universitaria, 10(2), 31-42.

Bacca, J., Baldiris, S., Fabregat, R., Graf, S., y Kinshuk, G. (2014): Augmented Reality Trends in Education: A Systematic Review of Research and Applications. Educational Technology \& Society, 17(4), 133-149.

Barba, R., Yasaca, S., y Manosalvas, C. (2015): Impacto de la realidad aumentada móvil en el proceso enseñanza-aprendizaje de estudiantes universitarios del área de medicina. En Adipe (Ed.), Investigar con y para la Sociedad, 3, (1421-1429). Cádiz, España: Bubok Publishing S.L.

Barroso, J., y Cabero, J. (2016). Evaluación de objetos de aprendizaje en realidad aumentada: estudio piloto en el grado de Medicina. Enseñanza \& Teaching, 34(2), 149-167.

Barroso, J., y Gallego, O. (2017). Producción de recursos de aprendizaje apoyados en Realidad Aumentada por parte de los estudiantes de magisterio. Edmetic. Revista de Educación Mediática y TIC, 6(1), 23-38.

Bates, T. (2015). Virtual Reality and education: some thoughts. Online learning and distance education resources. Recuperado de https://www. tonybates.ca/2017/07/27/virtual-realityand-education-some-thoughts

Bicen, H., y Bal, E. (2016). Determination of student opinions in augmented reality. Determination of student opinions in augmented reality. World Journal on Educational Technology: Current Issues, 8(3), 205-209, doi: https://doi. org/10.18844/wjet.v8i3.642

Bower, M., Howe, C., McCredie, N., y Robinson, A. (2014). Augmented Reality in education-cases, places and potentials. Educational Media International, 51(1), 1-15. doi: 10.1109/CICEM.2013.6820176.

Brigham, T. (2017). Reality Check: Basics of Augmented, Virtual, and Mixed Reality. Medical Reference Services Quarterly, 36(2), 171-178. doi: 10.1080/02763869.2017.1293987.

Brudniy, A., y Demilhanova, A. (2012). The Virtual Reality in a Context of the "Mirror Stage". International Journal of Advances in Psychology, 1, 6-9.

Cabero, J. (2016). ¿Qué debemos aprender de las pasadas investigaciones en Tecnología Educativa? Revista Interuniversitaria de Investigación en Tecnología Educativa (RIITE), 0, 23-33, doi: http://dx.doi. $\operatorname{org} / 10.6018 /$ riite/2016/256741

Cabero, J., y Barroso, J. (2016). The educational possibilities of Augmented Reality. NAER. New Approaches in Educational Research, 5(1), 44-50.

Cabero, J., y García, F. (Coords.) (2016). Realidad aumentada. Tecnología para la formación. Madrid: Síntesis.

Cabero, J., García, F., y Arroyo, C. (2016). La producción de objetos de aprendizaje en realidad aumentada para la formación universitaria en el SAV de la Universidad 
de Sevilla. En L. Villalustre y M. E. Del Moral (Eds.), Experiencias interactivas con realidad aumentada en las aulas (1930). Barcelona, España: Octaedro.

Cabero, J., López, E., y Jaén, A. (2013). Los portafolios educativos virtuales en las aulas universitarias: instrumentos didácticos para la innovación docente y la calidad de los procesos de enseñanzaaprendizaje. Enseñanza \& Teaching, 31, 45-70.

Cantón, D., Arellano, J., Hernández, M. A., y Nieva, O. (2017). Uso didáctico de la realidad virtual inmersiva con interacción natural de usuario enfocada a la inspección de aerogeneradores. Apertura, 9(2), 8-23.

Cañellas, A. (2017). Apuntes docentes: posibilidades educativas de la Realidad Virtual inmersiva. Centro de Comunicación y Pedagogía. Recuperado de http://www.centrocp.com/apuntesdocentes-posibilidades-educativas-larealidad-virtual-inmersiva/

Carbonell, C., y Bermejo, L. (2017). Landscape interpretation with augmented reality and maps to improve spatial orientation skill. Journal of Geography in Higher Education, 41(1), 119-133. doi: 10.1080/03098265.2016.1260530.

Castells, M. (2009). Acto de Investitura como Doctor Honoris Causa de la Universidad de Sevilla del profesor Dr. Manuel Castells Oliván. Sevilla: Secretariado de Publicaciones de la Universidad de Sevilla. Chang, H., Wu, K., y Hsu, Y. (2013). Integrating a mobile augmented reality activity to contextualize student learning of a socioscientific issue. British Journal of Educational Technology, 44, 3. doi: 10.1111/j.1467-8535.2012.01379.x.

Chang, X., Zhang, D., y Jin, X. (2016). Application of Virtual Reality Technology in Distance Learning. iJET, 11(11), 7679. doi: https://doi.org/10.3991/ijet. v11i11.6257

Chen, C. M., y Tsai, Y. (2012). Interactive augmented reality system for enhancing library instruction in elementary schools. Computers \& Education, 59, 638-652.

Cheng, K. H. (2017). Reading an augmented reality book: An exploration of learners' cognitive load, motivation, and attitudes. Australasian Journal of Educational Technologu, 33(4), 53-69. doi: https:/ doi.org/10.14742/ajet.2820

Cheng, Y., Lou, S., Kuo, S., y Shih, R. (2013). Investigating elementary school students' technology acceptance by applying digital game-based learning to environmental education. Australasian Journal of Educational Technology, 29(1). 96-110.

Chiang, T., Yang, S., y Hwang, G. (2014). Students' online interactive patterns in augmented reality-based inquiry activities. Computers \& Education, 78, 97-108. doi: http://dx.doi.org/10.1016/j. compedu.2014.05.006

Christensen, C. (2012). Disruptive innovation. En M. Soegaard y R. F. Dam (Eds.), Encyclopedia of humancomputer interaction. Aarhus, Denmark: The Interaction-Design.org Foundation. Recuperado de https://www. interaction-design.org/literature/book/ the-encyclopedia-of-human-computerinteraction-2nd-ed/disruptive-innovation

Cuendet, S., Bonnard, Q., Do-Lenh, S., y Dillenbourg, P. (2013). Designing augmented reality for the classroom. Computers \& Education 68, 5.57-569. doi: $\quad$ http://dx.doi.org/10.1016/i. compedu.2013.02.015

Cupani, M. (2012). Análisis de Ecuaciones Estructurales: conceptos, etapas de desarrollo y un ejemplo de aplicación. Revista Tesis, 1, 186-199.

Del Cerro-Velázquez, F., y Morales-Méndez, G. (2017). Realidad Aumentada como herramienta de mejora de la inteligencia espacial en estudiantes de educación secundaria. RED. Revista de Educación a Distancia, 53, 1-14. doi: http://dx.doi. $\mathrm{org} / 10.6018 / \mathrm{red} / 54 / 5$ 
Di Serio, A., Ibáñez, M. B., y Delgado, C. (2013): Impact of an augmented reality system on students' motivation for a visual art course. Computers \& Education, 68, 586-596. doi: 10.1016/j. compedu.2012.03.002.

Díaz, R. (2016). Augmented Reality Versus Virtual Reality: The Battle Is Real. Techcrunch. Recuperado de http:/ techcrunch.com/2016/01/04/ar-vs-vrthe-battle-is-real/?ncid=rss

Durall, E., Gros, B., Maina, M., Johnson, L., y Adams, S. (2012): Perspectivas tecnológicas: educación superior en Iberoamérica 2012-2017. Austin: The New Media Consortium.

Esteve-González, V., González, J., Gisbert, M., y Cela, J. M. (2017). La presencia social en entornos virtuales $3 \mathrm{~d}$ : reflexiones a partir de una experiencia en la Universidad. Pixel-Bit. Revista de Medios y Educación, 5O, 137-142. doi: 10.12795/pixelbit.2017. i50.09.

Ferrer, J., Jiménez, M. A., Torralba, J., y García, M. (2016). La realidad aumentada. Nuevas tecnologías en la formación de graduados en podología. Desarrollo y evaluación. En A. Allueva y J. L. Alejandre (Coords.), Simbiosis del aprendizaje con las tecnologías (147-160), Zaragoza, España: Prensa de la Universidad de Zaragoza.

Flores, J., Camarena, P., y Avalos, E. (2014). La Realidad Virtual una Tecnología Innovadora Aplicable al Proceso de Enseñanza de los Estudiantes de Ingeniería. Apertura. Revista de Innovación Educativa, 6(2), 86-99.

Fonseca, D., Redondo, E., y Valls, F. (2016): Motivación y mejora académica utilizando realidad aumentada para el estudio de modelos tridimensionales arquitectónicos. Education in the Knowledge Society, EKS, 17(1), 45-64.

Fowler, C. (2015). Virtual reality and learning: Where is the pedagogy? British
Journal of Educational Technology. 46(2), 412-422. doi: 10.1111/bjet.12135.

Freina, L., y Ott, M. (2015). A literature review on immersive virtual reality in education: State of the art and perspectives. Presentado en eLSE Conference, Bucharest. Recuperado de https://www.researchgate.net publication/280566372 A Literature Review on Immersive Virtual Reality in Education State of The Art and Perspectives

Garay, U., Tejada, E., y Maiz, I. (2017). Valoración de objetos educativos enriquecidos con realidad aumentada: una experiencia con alumnado de máster universitario. Pixel-Bit. Revista de Medios y Educación, 5O, 19-31.

Gómez, J. (2014). El fenómeno MOOC y la universalidad de la cultura: las nuevas fronteras de la Educación Superior. Revista de Currículum y Formación del Profesorado, 18(1), 73-91.

Gómez, J. (Ed.) (2016). UNIVERSITIC 2016. Análisis de las TIC en las Universidades Españolas. Madrid: Crue Universidades Españolas.

Guo, H. (2016). Application of Virtual Reality Technology in Swimming Teaching. iJET, 11(11), 9-14. doi: https://doi.org/10.3991 ijet.v11i11.6246

Han, J., Jo, M., Hyun, E., y So, H. (2015). Examining young children's perception toward augmented reality-infused dramatic play. Education Technology Research Development, 63, 455-474.

Ho, L. H., Hung, C. L., y Chen, H. C. (2013). Using theoretical models to examine the acceptance behavior of mobile phone messaging to enhance parent-teacher interaction. Computers \& Education, 61, 105-114. doi: http://dx.doi.org/10.1016/i. compedu.2012.09.009

Hofmann, S., y Mosemghvdlishvili, L. (2014). Perceiving spaces through digital augmentation: An exploratory study of navigational augmented 
reality apps. Mobile Media \& Communication, 2(3), 265-280. doi: 10.1177/2050157914530700.

Hsu, Y., Lin, Y., y Yang, B. (2017). Impact of augmented reality lessons on students' STEM interest. Research and Practice in Technology Enhanced Learning, 12(2), 1-14. doi: 10.1186/s41039-016-0039-z.

Hwang, W., y Hu, S. (2013). Analysis of peer learning behaviors using multiple representations in virtual reality and their impacts on geometry problem solving. Computers \& Education, 62, 308319. doi: http://dx.doi.org/10.1016/i. compedu.2012.10.005

Jeřábek, T., Rambousek, V., y Wildová, R. (2014). Specifics of Visual Perception of The Augmented Reality in The Context of Education. Procedia-Social and Behavioral Sciences, 159, 598-604.

Jimeno-Morenilla, A., Sánchez-Romero, J. L., Mora-Mora, H., y Collo-Miralles, H. (2016). Using virtual reality for industrial design learning: a methodological proposal. Behaviour \& Information Technology, 35(11), 897-906. doi: 10.1080/0144929X.2016.1215525.

Johnson, L., y Adams, S. (2016). NMC Horizon Report: 2016 Higher Education Edition. Austin. Texas: The New Media Consortium.

Johnson, L., Adams Becker, S., Cummins, M., Estrada, V., Freeman, A., y Ludgate, H. (2013). NMC Horizon Report: 2013 Higher Education Edition. Austin, Texas: The New Media Consortium.

Johnson, L., Adams Becker, S., Estrada, V., Freeman, A. (2014). NMC Horizon Report: 2014 Higher Education Edition. Austin: The New Media Consortium.

Johnson, L., Adams, S., Cummins, M., Estrada, V., Freeman, A. y Hall, C. (2016). NMC Horizon Report: 2016 Higher Education Edition. Austin: The New Media Consortium.

Kim, H., y Ke, F. (2016). OpenSimSupported Virtual Learning Environment:
Transformative Content Representation, Facilitation, and Learning Activities. Journal of Educational Computing Research, 54(2), 147-172. doi: 10.1177/0735633115620197.

Kim. K., Hwang, J., y Zo, H. (2016): Understanding users' continuance intention toward smartphone augmented reality applications. Information Development, 32(2), 161-174.

Lee, I., Chen, Ch., y Chang, K. (2016). Augmented reality technology combined with three-dimensional holography to train the mental rotation ability of older adults. Computers in Human Behavior, 65. 488-500. doi: http://dx.doi. org/10.1016/j.chb.2016.09.014

Lin, T., Been-Lirn, H., Li, N., Wang, H., y Tsa, Ch. (2013). An investigation of learners' collaborative knowledge construction performances and behavior patterns in an augmented reality simulation system. Computers \& Education, 68, 314321. doi: http://dx.doi.org/10.1016/j. compedu.2013.05.011

Marciniak, R., y Gairín, J. (2017). Un modelo para la autoevaluación de la calidad de programas de educación universitaria virtual. RED. Revista de Educación a Distancia, 54(2). doi: http://dx.doi. $\mathrm{org} / 10.6018 / \mathrm{red} / 54 / 2$

Marín, V. (2017). The augmented reality in the educational sphere of student of degree in chilhood education. Case study. Pixel-Bit. Revista de Medios y Educación, 51,7-19. doi: http://dx.doi.org/10.12795 pixelbit.2017.i51.01

Martín-Gutiérrez, J., Fabiani, P., Benesova, W., Meneses, M.D., y Mora, C.E. (2015). Augmented reality to promote collaborative and autonomous learning in higher education. Computers in Human Behavior, 51, 752-761. doi: http://dx.doi. org/10.1016/j.chb.2014.11.093

Martín, S. (2011). Educación Aumentada: Realidad o Ficción. Blog CUED. Recuperado de http://goo.gl/w46mpA 
Merino, E., Cabello, J., y Merino, E. (2017). El teléfono móvil y los estudiantes universitarios: una aproximación a usos, conductas y percepciones. Pixel-Bit. Revista de Medios y Educación, 51, 81-96.

Milgram, P., y Kishino, F. (1994). A Taxonomy of Mixed Reality Visual Displays. IEICE. TRANS. INF. \& SYS, 77(12), 1321-1329.

Nadolny, L. (2016). Interactive print: The design of cognitive tasks in blended augmented reality and print documents. British Journal of Educational Technology, 48(3), 814-823. doi: 10.1111/ bjet.12462.

Nielsen, B., Brandt, H., y Swensen, H. (2016). Augmented Reality in science educationaffordances for student learning. NorDina, 12(2), 157-174.

Pedraza, L. E., y Valbuena, S. (2014). Plataforma móvil con realidad aumentada para la enseñanza de los cálculos. Ventana Informática, 30, 205-216.

Pérez-López, D. (2015). eJUNIOR: Sistema de Realidad Aumentada para el conocimiento del medio marino en educación primaria. Quid, 24, 35-42.

Rasimah, C., Ahmad, A., y Zaman, H. (2011). Evaluation of user acceptance of mixed reality technology. Australasian Journal of Educational Technology, 27, 13691387.

Reinoso, R. (2016). Realidad aumentada posibilidades y usos educativos. En S. Baldiris y otros (Eds.), Recursos Educativos Aumentados. Una oportunidad para la inclusión (8-29). Colombia, Sello Editorial Tecnológico Comfenalco.

Rodríguez, A., Naranjo, M., y Duque, N. (2016). Prueba de usabilidad y satisfacción en objetos de aprendizaje con Realidad Aumentada en aplicaciones móviles. En S. Baldiris y otros (Eds.), Recursos Educativos Aumentados Una oportunidad para la inclusión (56-65). Colombia, Sello Editorial Tecnológico Comfenalco.
Sabino, C. (2006). La investigación científica. En F.G. Arias (Ed.), El proyecto de investigación. Introducción a la metodología científica (21-33). CaracasVenezuela: Editorial Episteme.

Saidin, N., Halim, N., y Yahaya, N. (2015). A Review of Research on Augmented Reality in Education: Advantages and Applications. International Education Studies, 8(13), 1-8.

Santos, M., Wolde, A., Taketomi, T., Yamamoto, G., Rodrigo, M., Sandor, Ch., y Kato, H. (2016). Augmented reality as multimedia: the case for situated vocabulary learning. Research and Practice in Techology Enhanced Learning, 11(4), 1- 23.

Tarng, W., y Ou, K.-L. (2012). A study of campus butterfly ecology learning system based on augmented reality and mobile learning. In IEEE Seventh International Conference on Wireless, Mobile and Ubiquitous Technology in Education (WMUTE) (pp. 62-66). Takamatsu: IEEE. Tecnológico de Monterrey (2015). Reporte EduTrends. Radar de Innovación Educativa 2015. Monterrey: Tecnológico de Monterrey.

Tecnológico de Monterrey (2016). Reporte EduTrends. Radar de Innovación Educativa 2016. Monterrey: Tecnológico de Monterrey.

Tecnológico de Monterrey (2017). Reporte EduTrends. Radar de Innovación Educativa 2017. Monterrey: Tecnológico de Monterrey.

Walsh, C., Garg, A., Ng, S., Goyal, F., y Grover, S. (2017). Residents' perceptions of simulation as a clinical. Canadian Medical Education Journal, 8(1), 76-87.

Wang, X., Jeong, M., Love, P., y Kang, S.C. (2013). Augmented Reality in built environment: Classification and implications for future research. Automation in Construction, 32 , 1-13. doi: http://dx.doi.org/10.1016/i. autcon.2012.11.021 
Wu, H. S, Wen-Yu, S., Chang, H. Y., y Liang, J. (2013). Current status, opportunities and challenges of augmented reality in education. Computers \& Education, 62, 41-49.

\section{PERFIL ACADÉMICO Y PROFESIONAL DE LOS AUTORES}

Julio Cabero Almenara. Catedrático de Tecnología Educativa de la Universidad de Sevilla, es director del Secretariado de Recursos Audiovisuales y Nuevas Tecnologías de la Universidad de Sevilla. Edito de la revista internacional Pixel Bit, Revista de Medios y Educación. Sus líneas de investigación giran en torno a las tecnologías emergentes.

E-mail: cabero@us.es

\section{DIRECCIÓN DEL AUTOR}

Facultad de Ciencias de la Educación

Universidad de Sevilla

Calle Pirotecnia s/n

411018 (España)

Bárbara Fernández Robles. Licenciada en Pedagogía y Doctora en educación por la Universidad de Córdoba (España). Sus líneas de investigación giran en torno a las tecnologías emergentes y a su integración en educación.

E-mail: bfernandezrobles@gmail.com

\section{DIRECCIÓN DEL AUTOR}

Facultad de Humanidades y Ciencias Sociales

Universidad Isabel I

Calle Fernán González, 76

09003 (España)

Fecha de recepción del artículo: 17/10/2017

Fecha de aceptación del artículo: 10/12/2017

\section{Como citar este artículo:}

Cabero Almenara, J., y Fernández Robles, B. (2018). Las tecnologías digitales emergentes entran en la Universidad: RA y RV. RIED. Revista Iberoamericana de Educación a Distancia, 21(2), pp. 119-138. doi: http://dx.doi.org/10.5944 ried.21.2.20094 